Focused deposition of nanoparticles on polymer fi I m wi th an i mproved TSI - nanoparti cl e sampl er ( Nodel 3089)

\begin{tabular}{|l|l|}
\hline 著者 & $\begin{array}{l}\text { Fukumor i Kanat a, Hi gashi H denori, Nai to } \\
\text { Takaaki, Hama Naoya, Kum ta M ki o, Set o } \\
\text { Takaf um, Ot ani Yoshi o }\end{array}$ \\
\hline $\begin{array}{l}\text { j our nal or } \\
\text { publ i cat i on t i t l e }\end{array}$ & Aer osol Sci ence and Technol ogy \\
\hline vol une & 49 \\
\hline number & 6 \\
\hline page range & $363-370$ \\
\hline year & $2015-06-03$ \\
\hline URL & ht t p: //hdl . handl e. net /2297/48649 \\
\hline
\end{tabular}


Revised manuscript (AST-MS-2014-239)

\section{Focused Deposition of Nanoparticles on Polymer Film with an Improved TSI-Nanoparticle Sampler (Model 3089)}

Kanta Fukumori ${ }^{1,3}$, Hidenori Higashi ${ }^{1}{ }^{1, \dagger}$, Takaaki Naito ${ }^{1}$, Naoki Shiratori ${ }^{1}$, Naoya Hama ${ }^{2}$, Mikio Kumita ${ }^{1}$, Takafumi Seto ${ }^{1}$ and Yoshio Otani ${ }^{1}$

${ }^{1}$ Graduate School of Natural Science and Technology, Kanazawa University, Kakuma-machi, Kanazawa, Ishikawa 920-1192, Japan

${ }^{2}$ Project Planning \& Development Group, Business Administration Department, Tokyo Dylec Corp., 1 Naito-machi, Shinjuku, Tokyo 160-0014, Japan

${ }^{3}$ Technical Research \& Development Institute, Sanki Engineering Co., LTD., 8-1, Akashi-cho, Chuoh, Tokyo 100-0001, Japan

†E-mail: hhigashi@mail.kanazawa-u.ac.jp

Keywords: nanoparticle, deposition, charging, electrostatic focusing, sampler 


\section{Abstract}

A two-dimensional array of spots of deposited nanoparticles as small as $7 \times 7 \mu \mathrm{m}$ was fabricated on a polymer film using a modified commercial nanometer aerosol sampler (NAS; TSI-model 3089) coupled with a surface-discharge microplasma aerosol charger (SMAC). The charged aerosol particles were electrostatically focused by a metal mesh (electrically grounded) on the polymer film (insulator) and electrode $(3 \mathrm{kV})$. The effect of mesh geometry on the concentration ratio (focusing ratio $\times$ collection efficiency) was evaluated using monodisperse polystyrene latex particles with diameters of 48,100 , and $300 \mathrm{~nm}$. The electrostatic focusing effect was also analyzed by a numerical simulation of the electrostatic field. The two-dimensional patterning of nanoparticles is an effective method in concentrating particles for the subsequent observation and chemical analysis of aerosol particles. In our experiments, the SMAC-NAS system achieved a net concentration ratio of more than 20 times for 48 - and 100-nm particles, which would significantly shorten the aerosol-sampling time. The particle deposition patterns formed on a transparent polymer film may provide samples for analyzing the transmittance, luminescence, and other optical characteristics of deposited nanoparticles. 


\section{INTRODUCTION}

Electrostatic deposition of charged particles on a solid substrate is a widely used technique for sampling and analyzing aerosol particles such as electrostatic precipitators and aerosol samplers (Fierz et al., 2007). Nanoparticles (particles of less than $100 \mathrm{~nm}$ in diameter) generally require a rather long sampling time due to their low charging probability and small mass. Although an effective charging method such as corona discharge can attain a high charging efficiency for nanoparticles, it is always accompanied with significant loss of nanoparticles within the charger. As a workaround, commercialized nanometer aerosol samplers (for example, NAS; TSI model 3089) target the collection of nanoparticles with an original electrical charge after the electrical mobility classification.

Focused deposition techniques for nanoparticles on specific 'spots' on a substrate have been developed for fabricating various nanoparticle-based devices. Lim et al. (2012), for example, reported on the focused deposition of aerosol nanoparticles using a transfer technique with photoresist. They successfully fabricated deposition patterns as small as $0.9 \mu \mathrm{m}$ in diameter, a scale that permits use in nanoparticle-based devices.

Focused deposition by this technique is also effective for concentrating nanoparticles for the subsequent aerosol chemical analyses. Fierz et al. (2007), for example, developed a portable electrostatic sampler for the observation of aerosol particles with a transmission electron microscope (TEM). They used a sampling electrode as small as the TEM observation grid (ø3 $\mathrm{mm}$ ) to concentrate aerosol on a confined area. Judging from their TEM images, a surface coverage of around 1 to $10 \%$ of the total area might be sufficient for the stochastic analysis of particles. Here, the surface coverage ratio for TEM observation, $S_{\mathrm{d}}$, can be calculated as $S_{\mathrm{d}}=C \times Q \times \Delta t \times A_{\mathrm{p}} / A$, where $C$ is the aerosol concentration, $Q$ is the flow rate, $\Delta t$ is the sampling time, $A_{\mathrm{p}}$ is the particle cross sectional area, and $A$ is the deposition area. The estimated sampling time for $10 \%$-coverage of the TEM grid (ø3 mm) by a uniform deposition of $100-\mathrm{nm}$ particles is as long as about $6 \mathrm{hrs}$ (for the case 
of $C=10^{4}$ particles $/ \mathrm{cm}^{3}$ and $Q=1 \mathrm{~L} / \mathrm{min}$ ). Methods for the chemical analysis of nanoparticles such as optical transmittance and spectroscopy may require more surface coverage $\left(S_{\mathrm{d}}\right.$ of more than $100 \%$, at least), which requires a significantly long sampling time. The focused deposition of nanoparticles in micron scale thus offers great benefits by reducing the sampling time and enhancing the time resolution of the analysis.

In this study we developed a method to focus the deposition of nanoparticles using a commercial nanometer aerosol sampler (NAS; TSI-model 3089) with simple improvements, coupled with a surface-discharge microplasma aerosol charger (SMAC; Manirakiza et al., 2011) for high throughput aerosol charging. The charged aerosol flux was focused simply by placing a metal mesh (electrically grounded) on a polymer film (insulator) and electrode $(3 \mathrm{kV})$. In this paper, we describe the experimental setup and the results, and discuss the effects of the particle diameter and mesh geometry on the focused deposition of nanoparticles.

\section{IMPROVEMENT OF NAS-MODEL 3089}

Figure 1 shows the basic configuration of the improved substrate holder of the NAS. The substrate holder of the original NAS is composed of a metal electrode $(8 \mathrm{~mm}$ in diameter) surrounded by an insulator (40 $\mathrm{mm}$ outer diameter). In order to enhance the electric field from the nozzle to the substrate (global field), we placed an electrode with a larger diameter (metal steel plate [c]; $23 \mathrm{~mm}$ in diameter and $0.3 \mathrm{~mm}$ in thickness) on the original electrode [b]. A transparent polymer film [d] (PEN film, Teijin DuPont Films, Teonex ${ }^{\circledR}$, thickness $\left.100 \mu \mathrm{m}\right)$ is used as a substrate for particle deposition. To focus the electric field (local field), a stainless steel mesh (SUS mesh \#200 \#30; listed in Table 1) which is commercially available for screen printing is placed on the PEN film. The focal area can be adjusted by changing the SUS mesh of different openings from 97 to $510 \mu \mathrm{m}$ and wire diameters ranging from 30 to $340 \mu \mathrm{m}$. An SUS cover with a single $20-\mathrm{mm}$ diameter hole is placed on the SUS mesh [f]. This SUS cover serves to both concentrate the electric field to the center 
of the electrode (shown later) and allow electrical contact with the SUS mesh. The SUS mesh and holder are both electrically grounded (the same electrical potential as the casing). The small gap between the electrode and mesh $(100 \mu \mathrm{m})$ limits the maximum applied voltage to the electrode to 3 $\mathrm{kV}$. This voltage was found to be sufficient in fabricating the nanoparticle deposition patterns described later in this paper.

\section{$<$ Figure 1 >}

\section{<Table 1>}

The electrostatic field in the sampler was calculated by a conventional software (EStat, Field Precision LLC, Advanced Science Laboratory) to analyze the focusing effect. The simulation was conducted by numerically solving Poisson's equation with the two-dimensional finite element method. Figure 2 shows the typical calculation result for an electrostatic field in the vicinity of the electrode. As the figure shows, the PEN film (relative dielectric constant of 3 ) is placed between the metal electrode $(+3 \mathrm{kV})$ and mesh $(0 \mathrm{~V})$. The four circles in Figure 2 represent the cross section of the SUS mesh (opening of $97 \mu \mathrm{m}$, wire diameter of $30 \mu \mathrm{m}$, electrically grounded). The upper area of the film and mesh is free space (nitrogen, relative dielectric constant of 1) where the charged aerosol is to be transported. Figure 2 shows the lines of electric force (solid lines) and the electric field strength (color). As shown in this figure, the lines of electric force are successfully deflected from the metal mesh (grounded) and penetrate to the PEN film (insulator) toward the electrode $(+3 \mathrm{kV})$. A similar focusing effect and particle trajectory were also numerically calculated for a charged-up photoresist surface by You and Choi (2007). The charged particles are expected to follow these lines of force and deposit on the localized area in the mesh opening. The focusing efficiency is estimated by the width of the lines of force for the different mesh geometries. 


\section{<Figure 2>}

The electrically grounded mesh is effective in focusing the local electric field on the substrate, but it also suppresses the transport of the charged aerosol from the inlet nozzle to the substrate by reducing the strength of the 'global' electric field. Figure 3 shows a typical electric field calculated for the whole area of sampler under four different conditions: (a) the original configuration with the metal plate mounted (20 mm diameter) and polymer film, (b) with the modification of inlet nozzle, (c) with the metal cover, and (d) with both the metal cover and mesh (\#200 mesh with opening of 97 $\mu \mathrm{m}$, wire diameter of $30 \mu \mathrm{m})$. The calculation condition and method are the same as those in Figure 2, but the number of calculation mesh in the domain was increased from the one employed in Figure 2 (20,000 mesh) to 1,670,000 for Figure 3 (a) (c) and to 4,870,000 for Figure 3 (d) . The white regions in Figs. 3 are the regions where the electric field strength exceeds $1.0 \times 10^{5} \mathrm{~V} / \mathrm{m}$. The electric field shown in Figure 3 (a) spreads out over a fairly wide area towards the casing. The trend is the same in Figure 3 (b), even after the modification of inlet nozzle (inner diameter of $8 \mathrm{~mm}$, nozzle-to-plate distance of $5 \mathrm{~mm}$ ). When the electrode is covered with the electrically grounded metal holder with the $20 \mathrm{~mm}$ hole (see Figure 1 for detail), as shown in Figure 3 (c), most of the electric field is concentrated toward the electrode and the strength of the electric field is maintained (about $440 \mathrm{kV} / \mathrm{m}$ ). It is seen in Figure 3 (d) that the metal mesh cover on the polymer film creates a local focusing field (Figure 2), but it also leads to the reduction in 'global' electric field from the nozzle to the electrode (about $54 \mathrm{kV} / \mathrm{m}$ with a mesh \#200). Consequently, the mesh geometries should be carefully chosen to obtain a sufficient electric field for conveying the charged nanoparticles toward the electrode. In the next section we describe the experimental evaluation of this effect.

\section{<Figure 3>}




\section{EXPERIMENTAL}

The performance of improved NAS was experimentally investigated using polystyrene latex (PSL) standard particles. Figure 4 shows the experimental setup. Test particles (PSL; 48, 100, and $300 \mathrm{~nm}$ ) were generated by a Collison type atomizer and diffusion dryer. The 300-nm PSL particles were fluorescent under ultraviolet (UV) irradiation for visualization of deposition patterns. The test particles (48 and $100 \mathrm{~nm}$ ) were electrically neutralized with an ${ }^{241}$ Am neutralizer and then classified by a differential mobility analyzer (long DMA, TSI model 3081, Sheath flow rate was set to 15 L/min). The $300 \mathrm{~nm}$ PSL particles were used without the size classification. A surface-discharge microplasma aerosol charger (SMAC, Manirakiza et al., 2011) was used as an additional charging device for high-throughput unipolar aerosol charging to increase the efficiency of the particle deposition. Although Manirakiza et al. (2011) used SMAC to investigate the high-efficiency charging of nanoparticles of less than $10 \mathrm{~nm}$, we applied it for the multiple charging of larger PSL particles. In our preliminary experiments we attained an average number of 3 elementary charges for 48-nm particles and 8 elementary charges for $100-\mathrm{nm}$ particles, and accordingly expected to obtain electrical mobility of $3.33 \times 10^{-7}$ for $48-\mathrm{nm}$ particles and $2.34 \times 10^{-7} \mathrm{~m}^{2} /(\mathrm{s} \mathrm{V})$ for $100-\mathrm{nm}$ particles. The deposition chambers used in the present experiment were those shown in Figures 1, 2, and 3. The aerosol flow rate was fixed at $0.3 \mathrm{~L} / \mathrm{min}$ with a pump of the condensation particle counter $(\mathrm{CPC}$, TSI model 3775). The CPC also monitored the particle concentration with and without the voltage applied to NAS, in order to measure the collection efficiency, $E_{\text {coll }}$, on the substrate (PEN film). The typical deposition time was about $60 \mathrm{~min}$ and the aerosol concentration was in the order of $10^{3}$ particle $/ \mathrm{cm}^{3}$. After the particle collection, the size and shape of deposition patterns were observed by a scanning electron microscope (SEM, HITACHI S-4500). In the runs using the fluorescent PSL particles $(300 \mathrm{~nm})$, the deposited film were illuminated by UV light (wavelength $254 \mathrm{~nm}$ ) and the fluorescent patterns were recorded by a digital camera. As an additional experiment, we also changed 
the opening and wire diameter of the SUS mesh (Table 1). The effectiveness of the electrostatic focusing, $R_{\text {focus, }}$ was calculated by the area of particle deposition pattern, $A_{\text {dep }}$, and the corresponding mesh area, $A_{\text {mesh: }}$ :

$$
R_{\text {focus }}=A_{\text {mesh }} / A_{\text {dep }}=\left(\text { mesh opening }+d_{\mathrm{w}}\right)^{2} /(\text { pattern size })^{2}
$$

where $d_{\mathrm{w}}$ which denotes the diameter of wire. To evaluate the efficiency with which the particles were deposited in a concentrated pattern for the subsequent chemical analysis, we defined the following net concentration efficiency, $R_{\mathrm{conc}}$, as,

$$
R_{\text {conc }}=E_{\text {coll }} \times R_{\text {focus. }}
$$

\section{<Figure 4>}

\section{RESULTS AND DISCUSSION}

Figure 5 shows the typical scanning electron micrograph of surface of the PEN film after 60 -min deposition of 100-nm PSL particles using the SUS mesh \#200 with a $97 \mu \mathrm{m}$ opening and wire diameter of $30 \mu \mathrm{m}$. It is clearly seen in Figure 5 that the particles were deposited in four localized square spots, each of which is about $7 \mu \mathrm{m}$ in width. This deposition pattern was formed over the entire deposition area $(\varnothing 20 \mathrm{~mm})$ with some deviations in density of particle deposition. The focusing ratio, $R_{\text {focus, }}$ is equal to $330\left(=(97+30)^{2} / 7^{2}\right)$. Since the particle collection efficiency was $E_{\text {coll }}=0.10$, the net concentration efficiency $R_{\text {conc }}$ is $33(=0.10 \times 330)$. In total, 33 -fold concentration (sampling time reduction to $1 / 33$ ) was achieved with the improved NAS.

Figures 6 (a) to (f) show the deposition patterns of 48-nm and 300-nm PSL particles using three types of SUS meshes. These figures clearly show the regular deposition patterns on the PEN film. We also see in these figures that an increases in mesh opening leads to a larger deposition spot area and correspondingly a lower particle density. Furthermore, we may observe in Figure 6 that the shape of deposition spots changes from squares ((a) and (d)) to corner-rounded diamonds ((c) and 
(f)) as the mesh opening increases. The change in shape of deposition spot probably resulted from a deformation of the electrostatic field caused by the overwrapping of electrical fields at the intersections of wires.

\section{<Figure 5>}

\section{<Figure 6>}

In Figure 7, the measured spot size of particle deposition is plotted as a function of the mesh opening. Since the shape of deposition spots changed from squares to corner-rounded diamonds, the measured sizes are plotted with the error bars ( \pm 1 standard deviations of Felet diameters of spots). The spot size increases almost linearly with the mesh opening. The same tendency was also found for the different particle diameters and wire diameters studied in the present experiment. Table 2 summarizes the experimental conditions under which we were successful in creating deposition patterns. Note that we were not able to determine the collection efficiency of 300 -nm particles because of the generation of residual particles during the atomization.

Figure 8 shows the calculated width of the lines of electric force (focusing width) as a function of the mesh opening and wire diameter. The focusing width, $W$, was obtained by tracing the lines of electric force starting from the upstream to the film surface (shown in the inset of Figure 8). Figure 8 shows that the focusing width (spot size) decreases with reducing the mesh opening, as found in the experiment. In the simulation, the focusing effect was predicted to be more significant for the mesh with a thicker wire. In contrast, the measured pattern sizes (Figure 7) are larger than the sizes predicted by the simulation, and no clear tendency versus the wire diameter could be experimentally observed. We therefore speculate that the aerosol trajectory is affected by the other deposition mechanisms such as the diffusion, inertia, or local turbulence in the flow. We performed preliminary numerical simulation of particle trajectory using a conventional software, COMSOL Multiphysics ${ }^{\circledR}$ 
(COMSOL Inc.) with considering the electrostatic force, inertia and gas flow. As shown in supplement information (Figure S3), the focusing width calculated by particle trajectory were basically similar to those calculated from the width of the electric field (Fig. 8). Thus we may roughly estimate the optimum mesh geometry for the patterned deposition of a given size of particles.

\section{<Figure 7>}

\section{<Figure 8>}

\section{<Table 2>}

When we use this collection device for aerosol chemical analyses, we should also consider the net concentration efficiency. Table 2 lists the aerosol collection efficiency, $E_{\text {coll }}$, focusing ratio, $R_{\text {focus, }}$ and concentration ratio $\left(=R_{\text {focus }} \times E_{\text {coll }}\right)$ for all experimental conditions where particle deposition was observed. Smaller openings were preferable for the fabrication of smaller spots as shown in the Table, but they also lowered the collection efficiency because of the lower global electrostatic fields. Figure 9 shows the plots of the collection efficiency against the mesh opening ratio (=open area/total area). It shows that the collection efficiency is a function of the mesh opening ratio. A mesh opening ratio larger than $40 \%$ was required for the focused particle deposition, which corresponds to the electric field strength of the order of $10^{5} \mathrm{~V} / \mathrm{m}$ (Figure S1). Note that the collection efficiency was low (less than $25 \%$ ) under our experimental conditions because the electrostatic force was not enough strong to defy the aerosol flow.

<Figure 9 (a), (b)>

In summary, the mesh geometry (mesh opening and wire diameter) and deposition time for 
fabricating the desired deposition pattern should be carefully selected in consideration of the spot size and aerosol concentration required. As shown in Table 2, a smaller mesh opening increased the focusing ratio but decreased the collection efficiency. Therefore, \#200 mesh would be suitable for fabricating as small a spot as possible (less than $10 \mu \mathrm{m}$ ), but the collection concentration efficiency with such a mesh would be less than $10 \%$. If one wishes to take advantage of the shorter deposition time for aerosol sampling rather than the spot size, or to target the collection of aerosol at an extremely low concentration, \#40B mesh would exhibit a higher collection efficiency than the other meshes because of the largest mesh opening ratio. In both applications, a focused aerosol deposition may contribute to a rapid and highly sensitive aerosol sampling.

The smallest pattern size attained in our experiments was $7 \mu \mathrm{m}$, as we were unable to find the condition for successful deposition with an opening of smaller than $97 \mu \mathrm{m}$. Lee et al. (2011) and Lim et al. (2012) were successful in fabricating a sub-micron pattern using a special pretreatment (nanoxerography technique). We believe, however, that the pattern size in our simple system may suffice the concentrating aerosol particles for chemical analysis. Chemical analysis methods such as micro-Raman spectroscopy are typically performed with laser beams of only a few microns in diameter (Guedes et al. 2008), and laser-induced breakdown spectroscopy is performed with a laser beam of a 100- $\mu \mathrm{m}$ in diameter (LIBS, Park et al., 2009). A key advantage of our system for those optically-probed analyses is its ability to deposit particles on a transparent polymer film. As an example, two-dimensional array of deposited patterns composed of fluorescent PSL particles (300 $\mathrm{nm}$ ) illuminated by UV light was shown in Figure S3 in the supplement information.

\section{SUMMARY}

Our group achieved the focused deposition of charged particles on a polymer film using a commercial nanometer aerosol sampler (NAS Model 3089) with simple modifications. We analyzed the electrostatic focusing effect by numerically calculating the electrostatic field. An SUS mesh 
integrated with the sampler proved to be successful in both fabricating a uniform two-dimensional pattern of deposited nanoparticles with diameters as small as $7 \mu \mathrm{m}$ and in concentrating aerosol particles by more than 20 times. We expect this new technique to be applied to various systems for rapid and highly sensitive aerosol sampling and analysis.

\section{ACKNOWLEDGEMENT}

We are grateful to Teijin DuPont Films for supplying PEN film, Teonex ${ }^{\circledR}$.

\section{REFERENCES}

Fierz, M., Kaegi, R. and Burtscher, H. (2007). Theoretical and Experimental Evaluation of a Portable Electrostatic TEM Sampler. Aerosol Sci. Technol. 41(5): 520-528.

Guedes, A., Valentim, B., Prieto, A. C., Sanz, A., Flores, D. and Noronha, F. (2008). Characterization of Fly Ash From a Power Plant and Surroundings by Micro-Raman Spectroscopy. Int. J. Coal Geology. 73: 359-370.

Lee, H., You, S., Pikhitsa, P. V., Kim, J., Kwon, S., Woo C. G. and Choi, M. (2011). Three-Dimensional Assembly of Nanoparticles from Charged Aerosols. Nano Lett. 11: 119-124.

Lim, K., Lee, J. R., Lee, H., Pikhitsa, P. V., You, S., Woo, C. G., Kim, P., Suh, K. Y. and Choi, M. (2012). Nanoxerography Utilizing Bipolar Charge Patterns. Appl. Phys. Lett. 101: 203106.

Manirakiza E., Seto, T., Osone, S., Fukumori, K. and Otani Y. (2011). High-Efficiency Unipolar Charger for Sub-10 nm Aerosol Particles using Surface-Discharge Microplasma with a Voltage of Sinc Function. Aerosol Sci. Technol. 47: 60-68.

Park, K., Cho, G. and Kwak, J. H. (2009). Development of an Aerosol Focusing-Laser Induced Breakdown Spectroscopy (Aerosol Focusing-LIBS) for Determination of Fine and Ultrafine Metal Aerosols. Aerosol Sci. Technol. 43: 375-386.

You, S. and Choi, M. (2007). Numerical simulation of microscopic motion and deposition of 
nanoparticles via electrodynamic focusing. J. Aerosol Sci. 38: 1140-1149. 
Figure captions

FIG. 1 Basic design of the improved sampling holder for the NAS model 3089

FIG. 2 Calculated electric field near the surface of the polymer film (local field)

FIG. 3 Calculated electric field (global field)

FIG. 4 Schematic diagram of the experimental setup

FIG. 5 Micro array generated on the polymer film (PSL particles of 100-nm diameter)

FIG. 6 Scanning electron micrographs of nanoparticle deposition patterns: (a) to (c) $d_{\mathrm{p}}=48 \mathrm{~nm}$, (d) to (f) $d_{\mathrm{p}}=300 \mathrm{~nm}$. Mesh openings and wire diameters: (a), (d) $97 \mu \mathrm{m} / 30 \mu \mathrm{m}$ (\#200), (b), (e) $300 \mu \mathrm{m} /$ $120 \mu \mathrm{m}$ (\#60B), (c), (f) $500 \mu \mathrm{m} / 140 \mu \mathrm{m}$ (\#40B)

FIG. 7 Width of the nanoparticle pattern generated on the polymer film plotted against the size of the mesh opening

FIG. 8 Width of the lines of electric force plotted against the mesh opening

FIG. 9 Collection efficiency plotted against the mesh opening ratio 
Fig.1

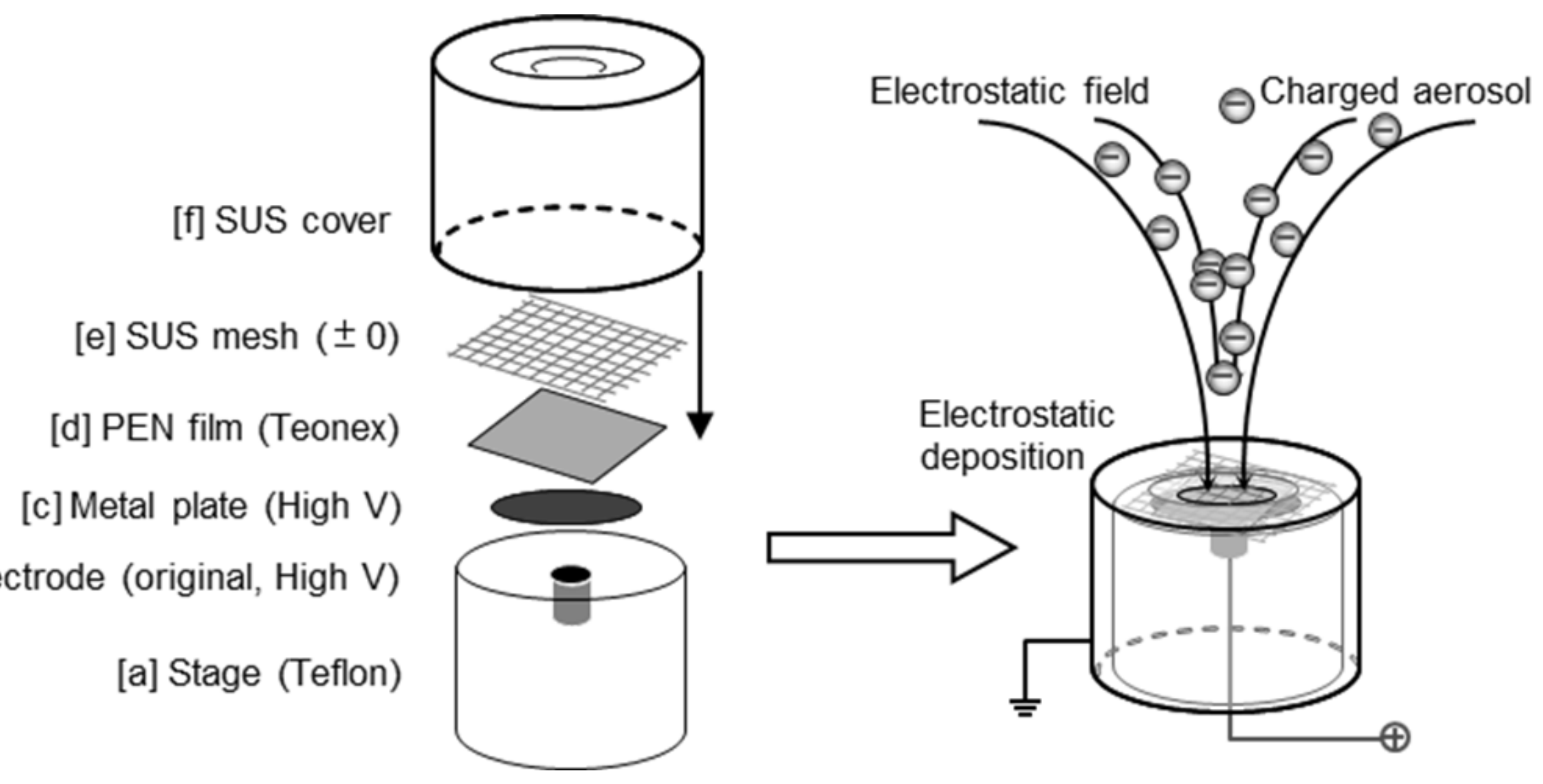

[b] Electrode (original, High V)

[a] Stage (Teflon) 
Fig.2

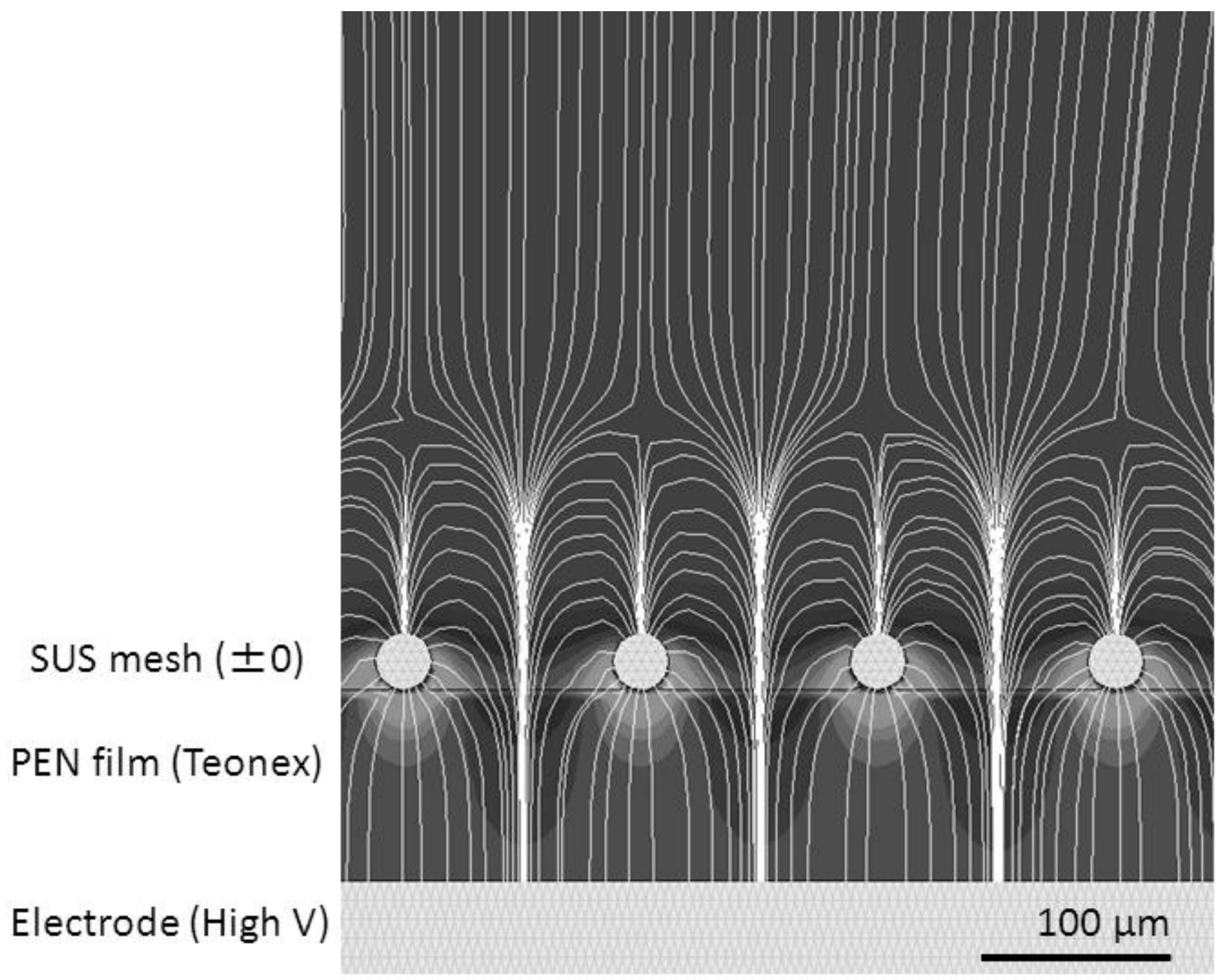

\section{Electric field Strength $[\mathrm{V} / \mathrm{m}]$}

$8.6765 \times 10^{5}$ $1.7353 \times 10^{5}$ $2.6029 \times 10^{5}$ $3.4706 \times 10^{5}$ $4.3382 \times 10^{5}$ $5.2059 \times 10^{5}$ $6.0735 \times 10^{5}$ $6.9412 \times 10^{5}$ $7.8088 \times 10^{5}$ $8.6765 \times 10^{5}$ $9.5441 \times 10^{5}$ $1.0412 \times 10^{6}$ 


\section{Fig.2 (color)}

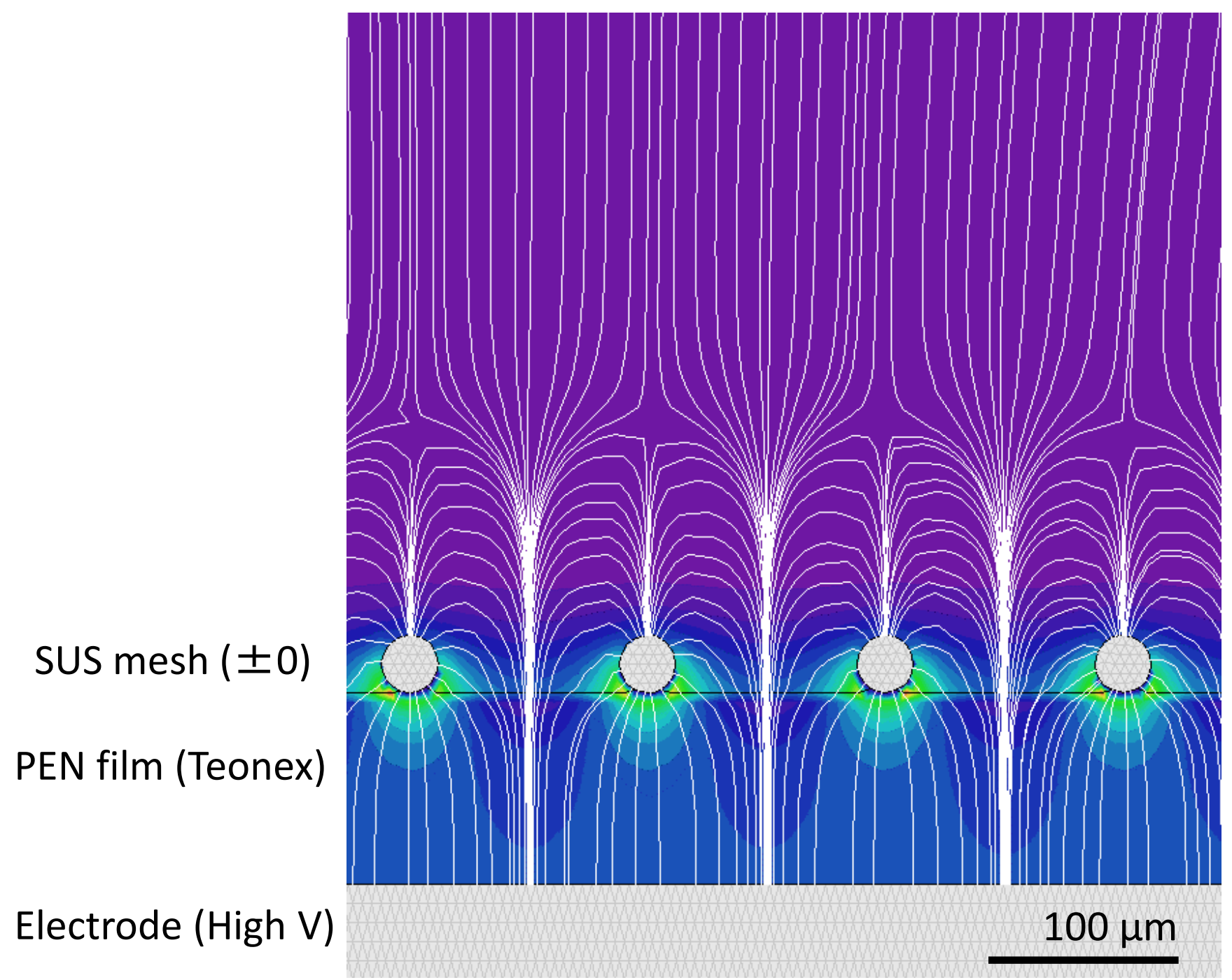

\section{Electric field Strength [V/m]}

$8.6765 \times 10^{5}$ $1.7353 \times 10^{5}$ $2.6029 \times 10^{5}$ $3.4706 \times 10^{5}$ $4.3382 \times 10^{5}$ $5.2059 \times 10^{5}$ $6.0735 \times 10^{5}$ $6.9412 \times 10^{5}$ $7.8088 \times 10^{5}$ $8.6765 \times 10^{5}$ $9.5441 \times 10^{5}$ $1.0412 \times 10^{6}$ 


\section{Fig.3}

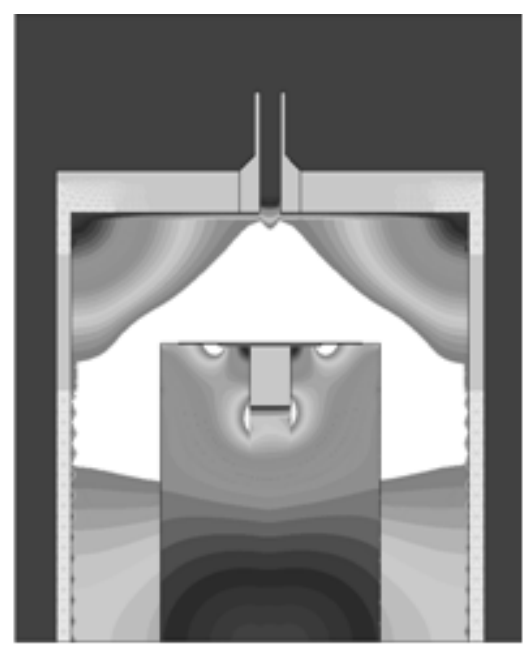

(a) Original with mounting metal plate

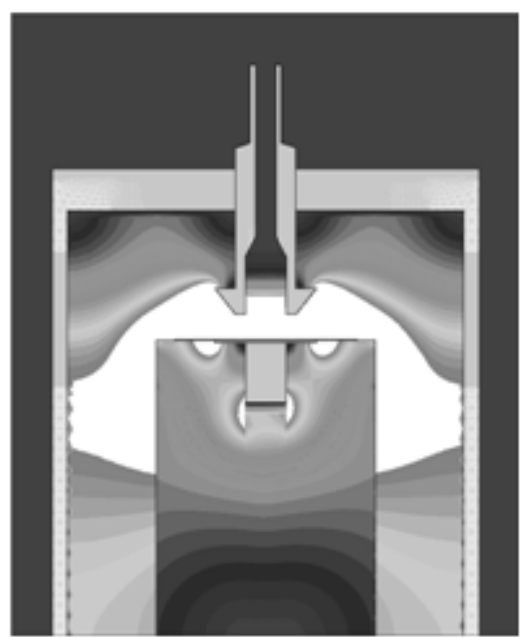

(b) Only film with modified nozzle

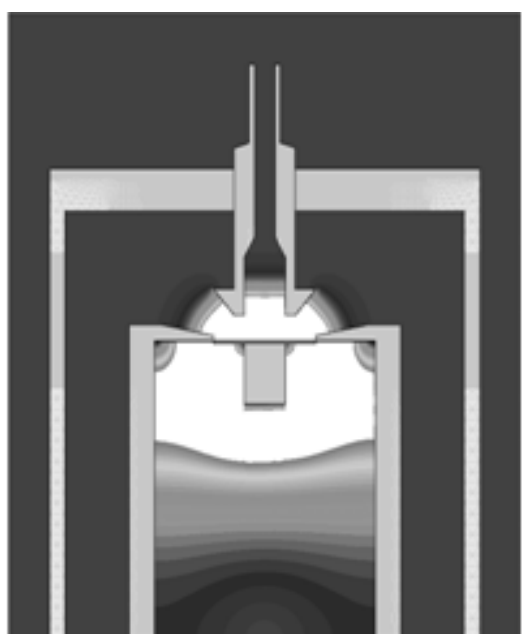

(c) With stainless steel cover

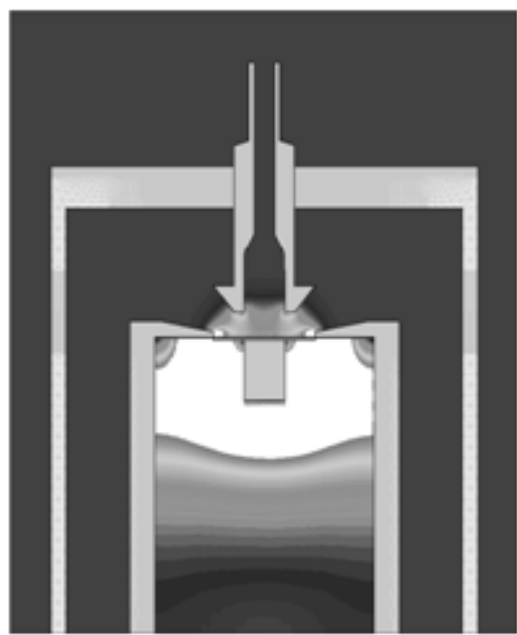

Electric field

strength $[\mathrm{V} / \mathrm{m}]$

$8.3333 \times 10^{3}$ $1.6667 \times 10^{4}$ $2.5000 \times 10^{4}$ $3.3333 \times 10^{4}$ $4.1667 \times 10^{4}$ $5.0000 \times 10^{4}$ $6.6667 \times 10^{4}$ $7.5000 \times 10^{4}$ $8.3333 \times 10^{4}$ $9.1667 \times 10^{4}$ $1.0000 \times 10^{5}$

(d) With stainless steel mesh 


\section{Fig.3 (color)}

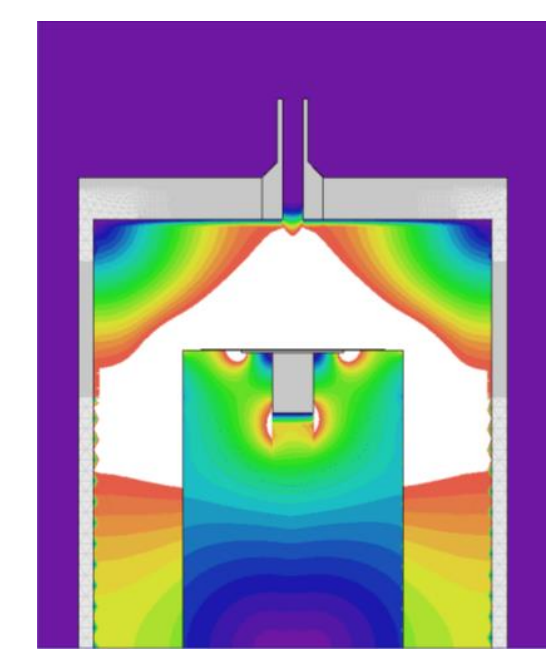

(a) Original with mounting metal plate

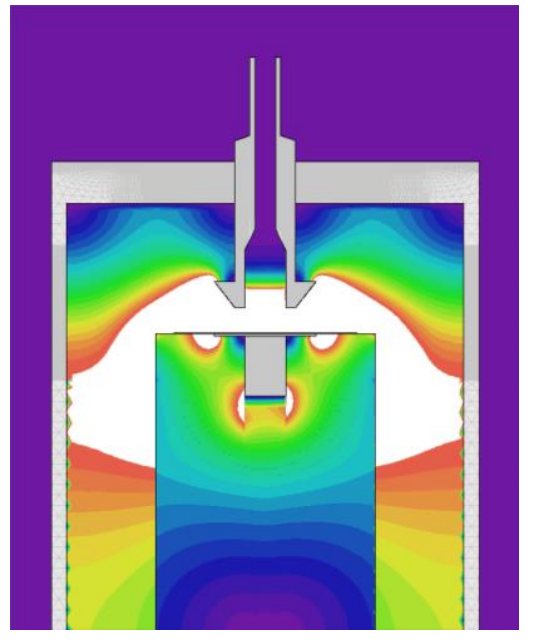

(b) Only film with modified nozzle

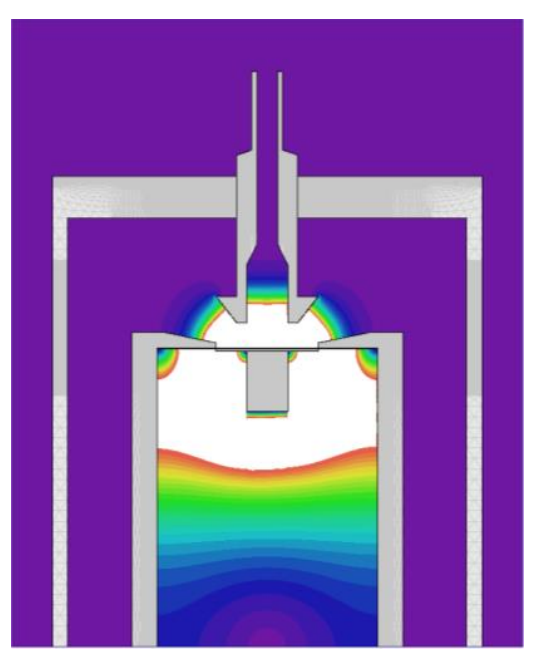

(c) With stainless steel cover

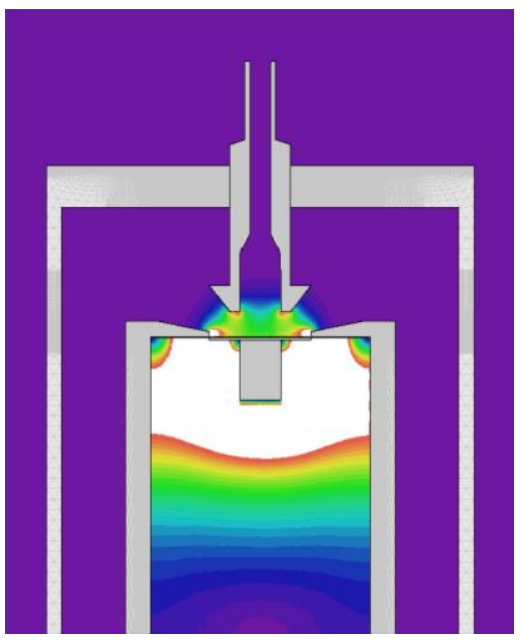

Electric field

strength $[\mathrm{V} / \mathrm{m}]$

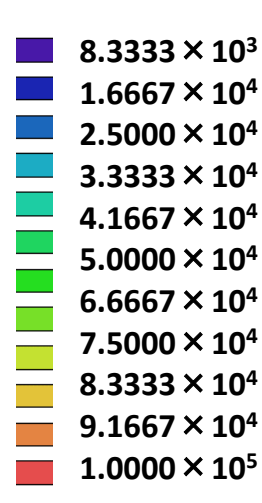

(d) With stainless steel mesh 
Fig.4

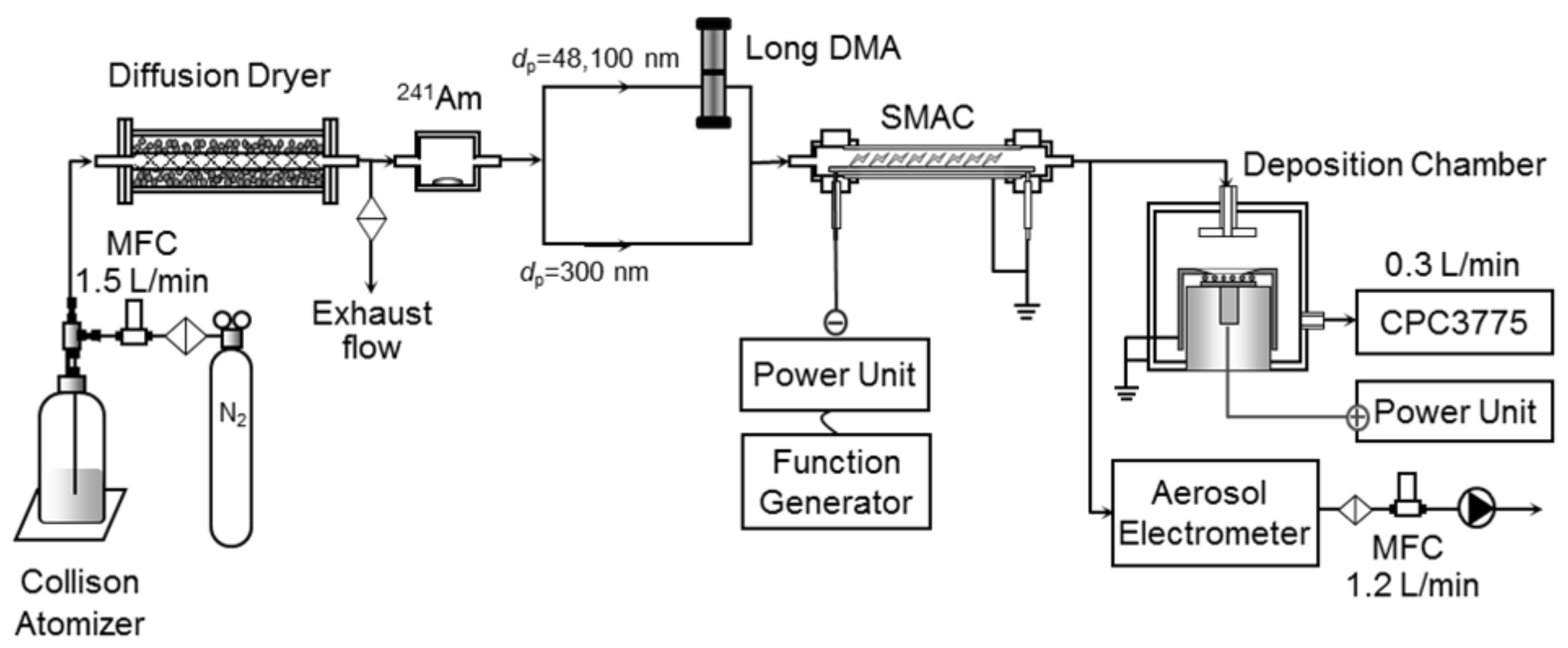


Fig.5
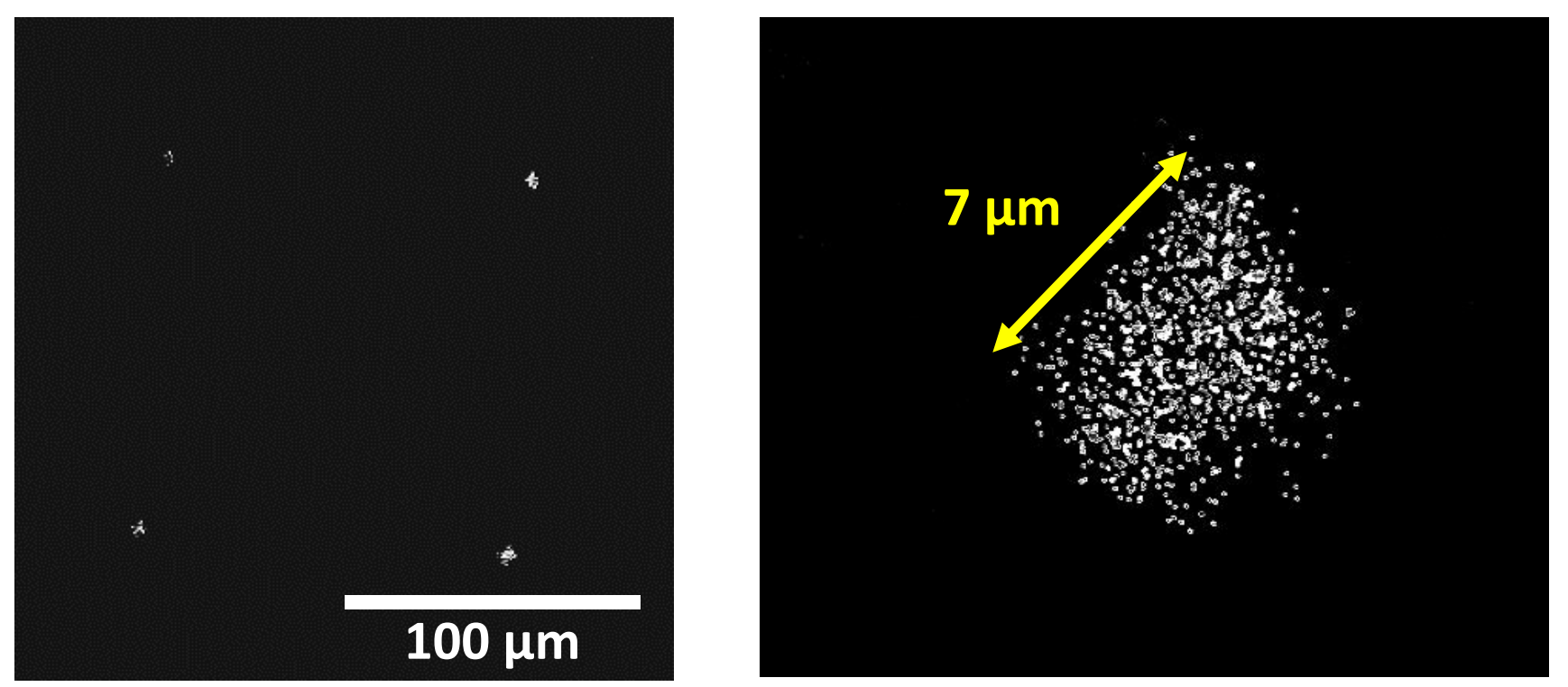
Fig.6

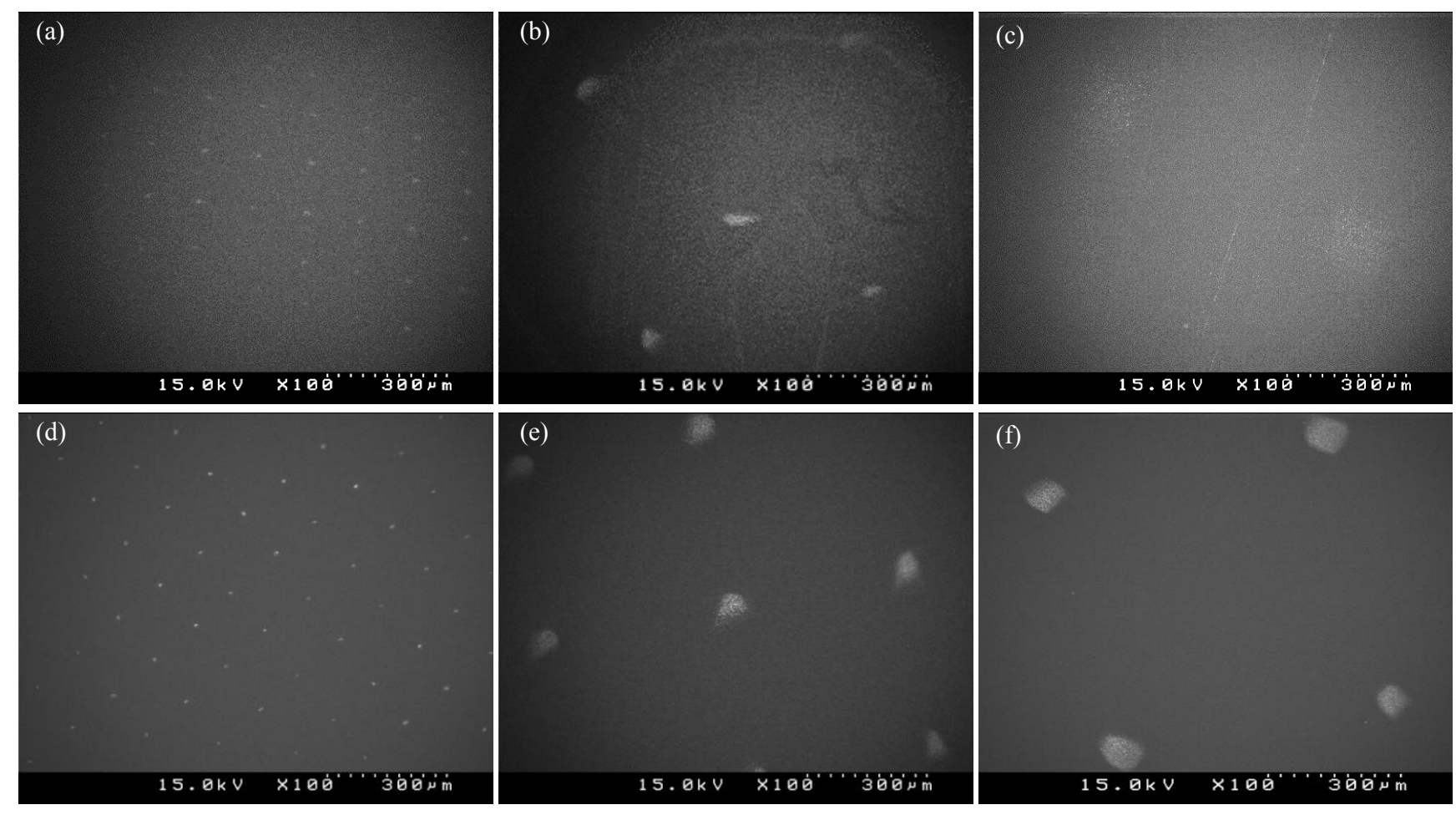


Fig.7

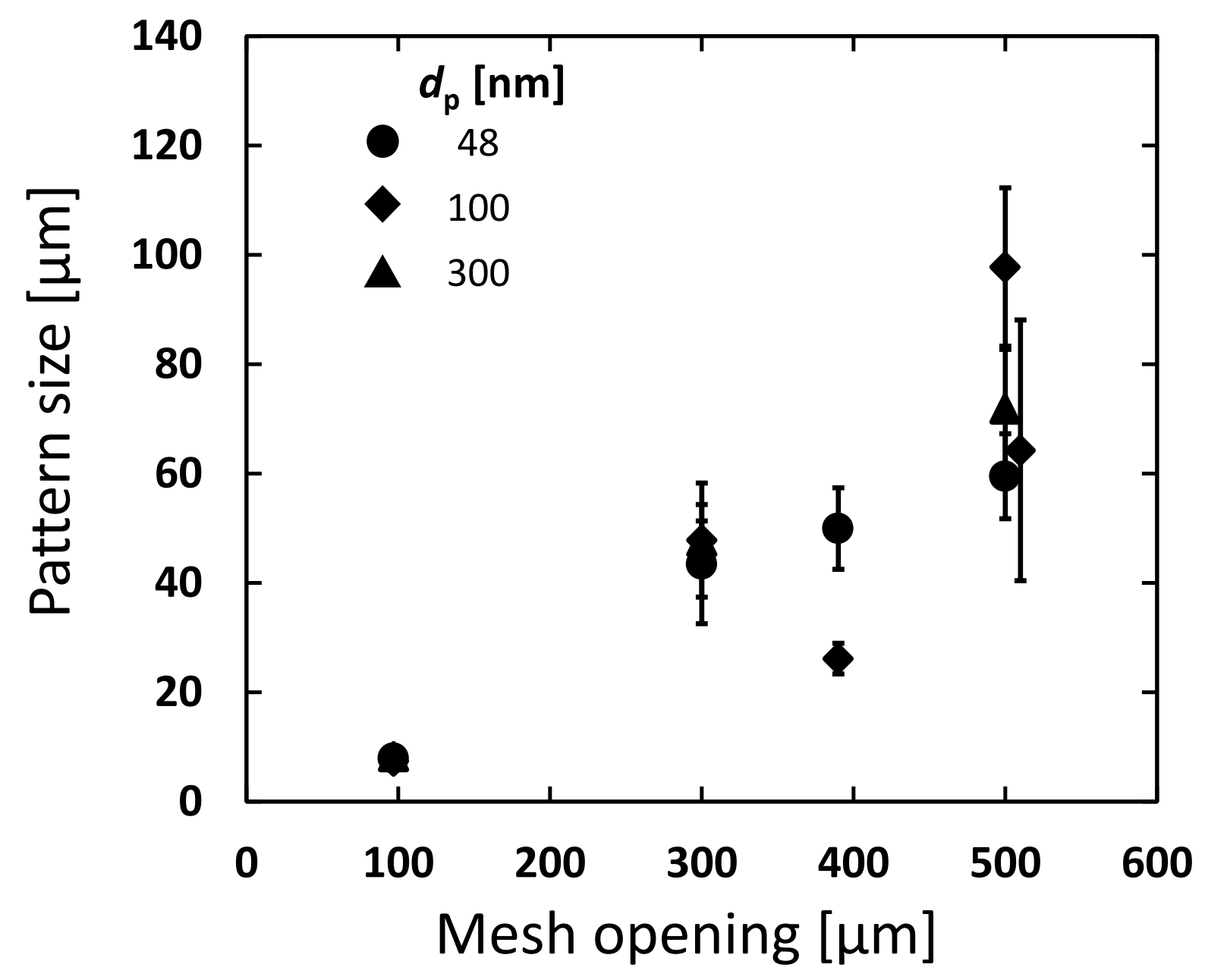


Fig.8

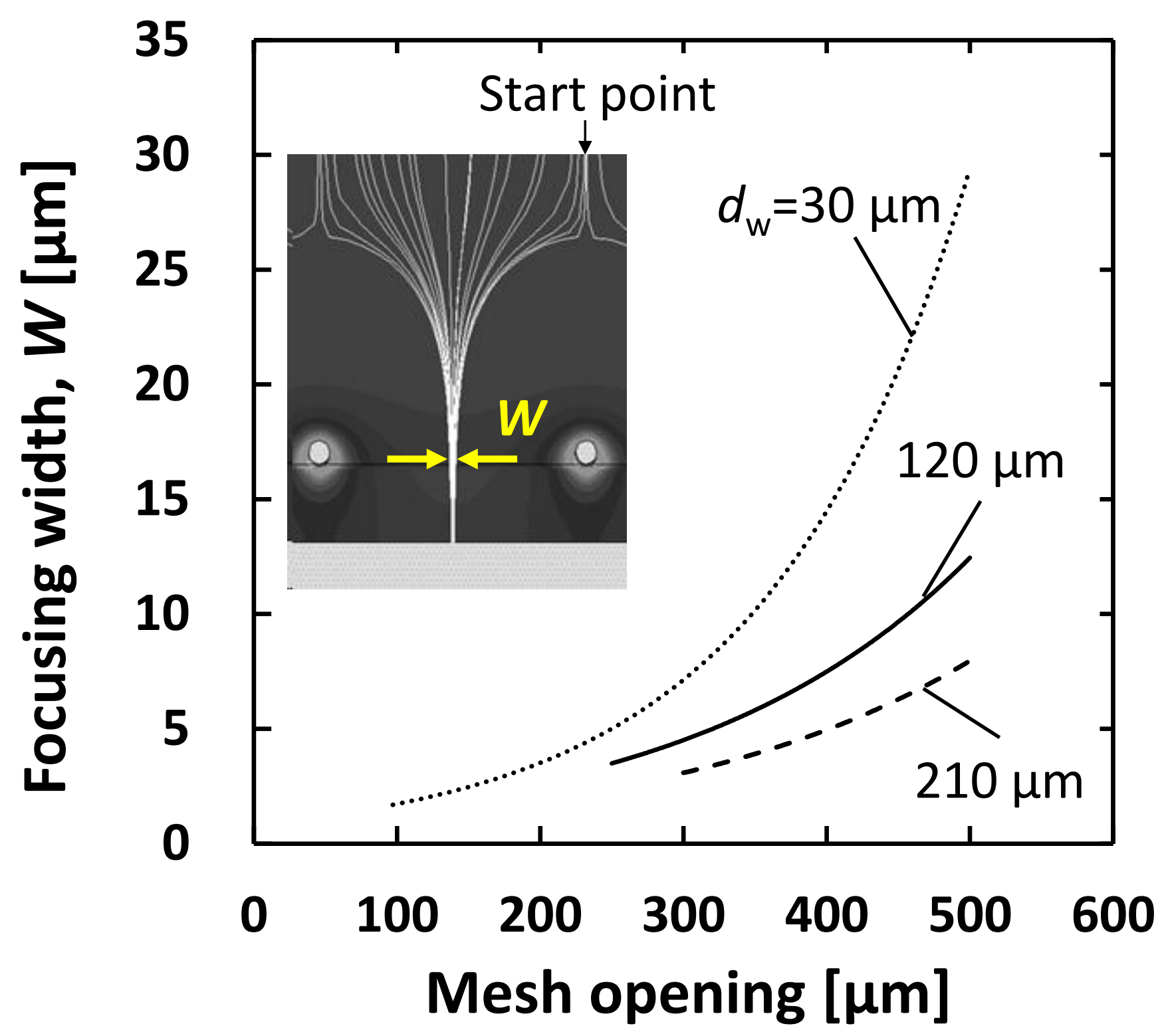


Fig.8 (color)

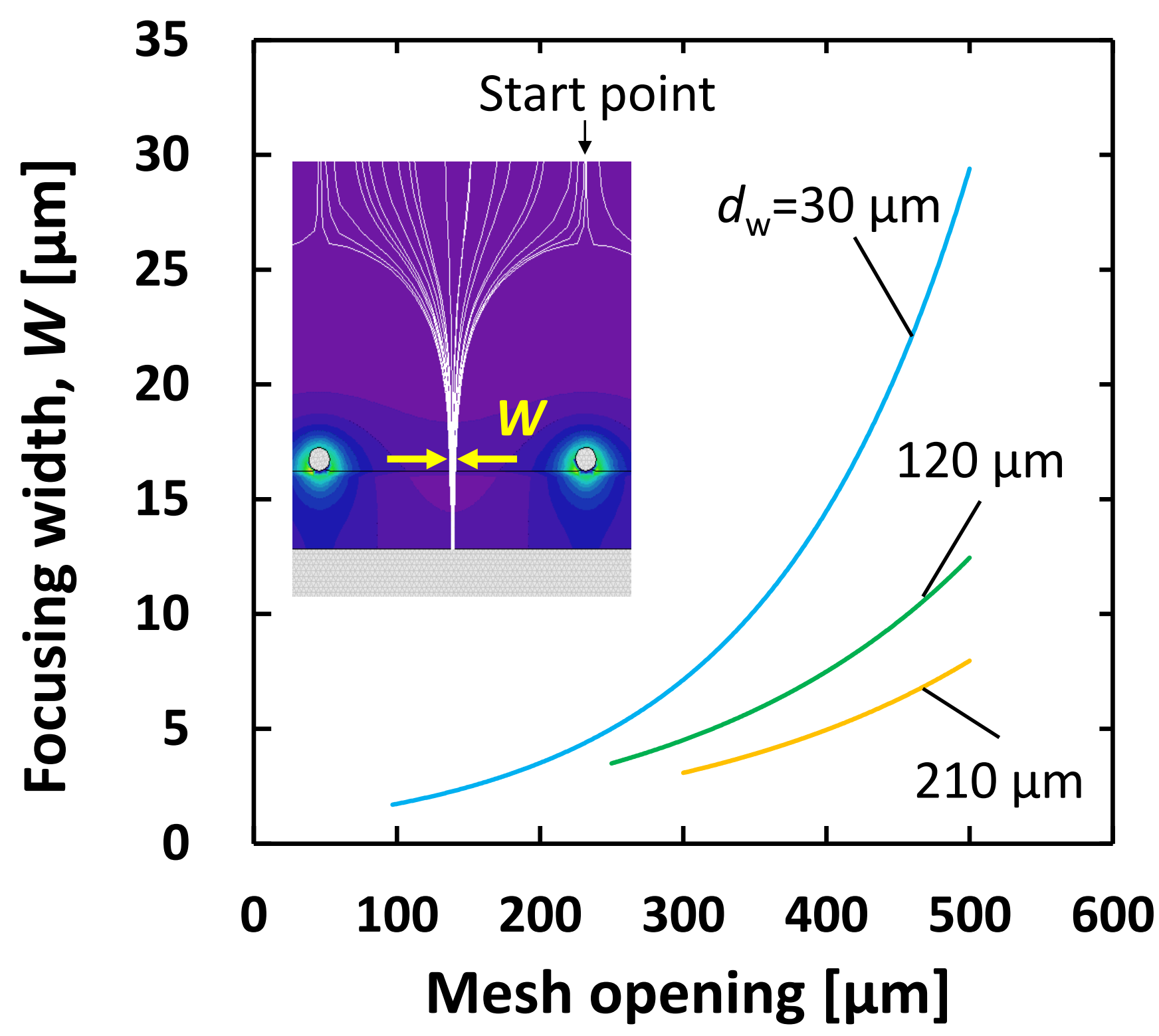


Fig.9

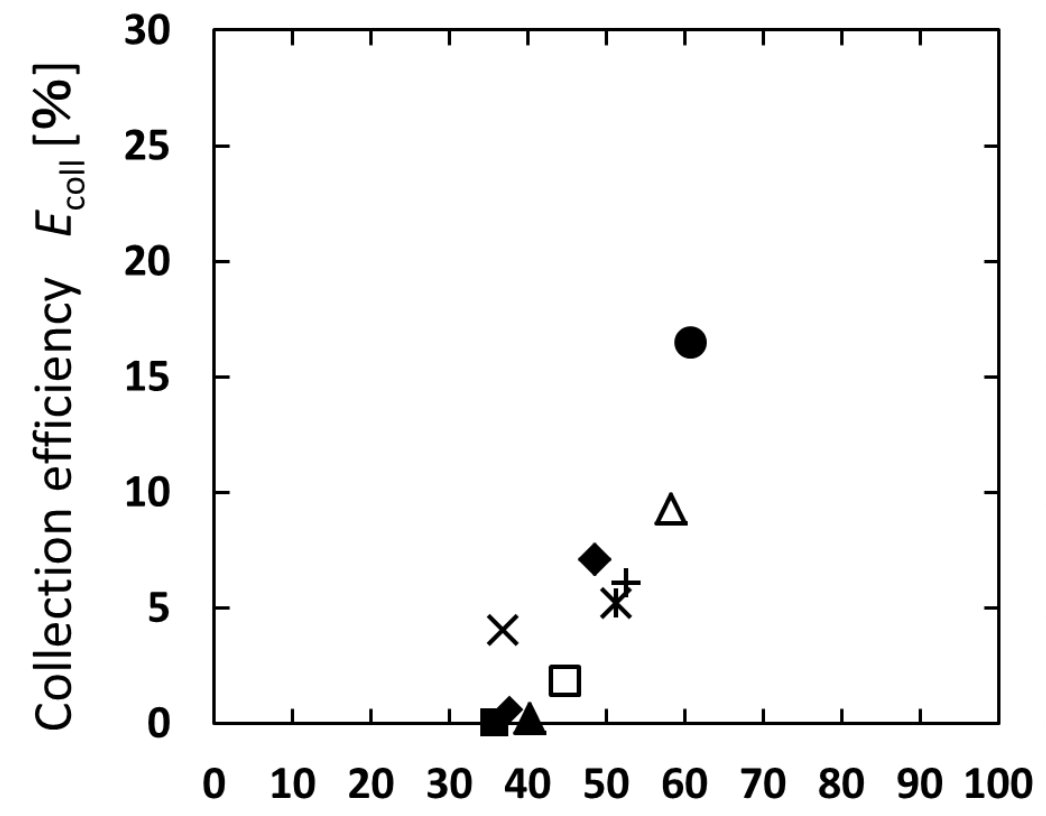

Mesh opening ratio [\%]
Mesh number/

Mesh opening / Wire diameter $[\mu \mathrm{m}]$

$\Delta \# 200 / 97 / 30$

- \#70/220/140

口\#60/280/140

* \#60B/300/120

$+\# 50 / 370 / 140$

$\times \# 40 / 390 / 250$

- \#40B/500/140

४\#35/510/220

$\Delta \# 32 / 500 / 290$

- \#30/510/340

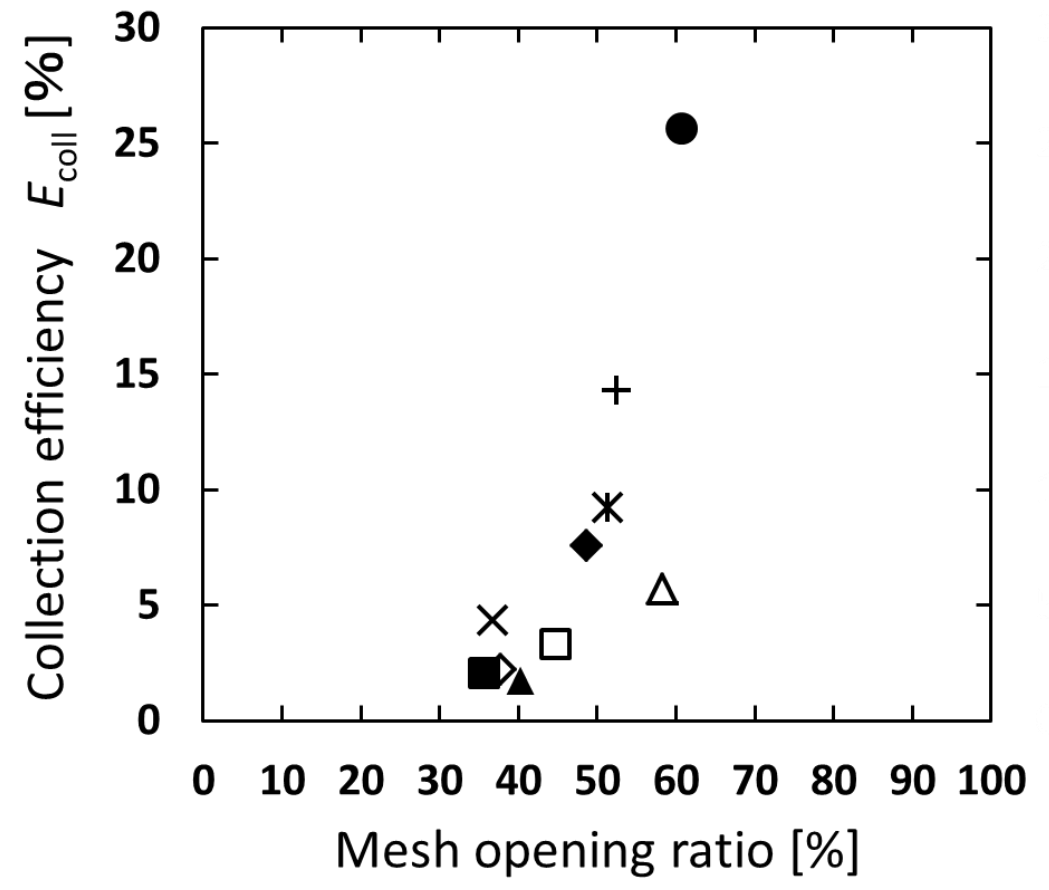

(b) $d_{\mathrm{p}}=100 \mathrm{~nm}$ 